Subject strapline: Environmental microbiology

\title{
Cryptic microbial hydrocarbon cycling
}

Terry J. McGenity ${ }^{1 *}$, Boyd A. McKew ${ }^{1}$, David J. Lea-Smith ${ }^{2}$

${ }^{1}$ School of Life Sciences, University of Essex, Wivenhoe Park, Colchester, CO4 3SQ. UK

${ }^{2}$ School of Biological Sciences, University of East Anglia, Norwich Research Park, Norwich, NR4 7TJ. UK

* Corresponding Author: Terry J. McGenity, School of Life Sciences, University of Essex, Wivenhoe Park, Colchester, CO4 3SQ. United Kingdom. Telephone +44 1206 872535. Email timcgen@essex.ac.uk

\section{Standfirst}

Cyanobacteria, which produce $\sim 25 \%$ of global oxygen, also release around 100 times more hydrocarbons into the ocean than all petroleum sources. A cryptic microbial cycle explains why these hydrocarbons do not accumulate in the sea.

\section{Main text}

Petroleum is formed over millions of years and contains thousands of structurally diverse hydrocarbons. These are widespread in the ocean, with natural oil seeps and human activities releasing between 0.47 and 8.3 million tonnes of petroleum annually [1]. Microbial degradation of the chemicals in petroleum is a critical step in this long-term hydrocarbon cycle (Fig. 1). Although it has been known for decades that phytoplankton are also a source of hydrocarbons such as alkanes and alkenes, they were not accounted for in any marine hydrocarbon census. Previous work measuring hydrocarbon production in laboratory cultures of Prochlorococcus and Synechococcus, the two most prevalent marine cyanobacteria, estimated that they produce between 300 and 800 million tonnes of alkanes per year (primarily the 15-carbon, straightchain alkane, $n$-pentadecane), based on their abundance and turnover in the ocean; approximately 100 -fold greater than petroleum released from seeps and anthropogenic 
discharges combined [2]. In the current issue of Nature Microbiology, Love et al. [3] use in-situ measurements and scale-up models to assess whether marine cyanobacteria produce this much hydrocarbon in the ocean and how this might impact ecosystem functioning [2]. The authors estimated that global cyanobacterial hydrocarbon production rates range from 118 to 649 million tonnes per year or $\sim 100-500$ fold more than petroleum inputs [3], which is in broad agreement with the projections made from laboratory cultures [2].

In their extensive and multifaceted field study, Love et al. [3] measured alkanes in the particulate fraction of 441 samples from North Atlantic waters that were mostly oligotrophic. This region is representative of $\sim 40 \%$ of the global ocean where Prochlorococcus and Synechococcus are the dominant photoautotrophs. The particulate fraction containing microbial cells was studied because alkanes are found in cyanobacterial membranes, where they are essential for optimising cell size and division [4]. The predominantly detected alkane in situ was $n$-pentadecane, and its concentration along depth profiles correlated with cyanobacteria cell counts, peaking in the deep chlorophyll maximum. Thus, cyanobacteria were identified as the primary candidates for $n$-pentadecane production.

Using shipboard incubations with ${ }^{13} \mathrm{C}$-labelled bicarbonate as a source of dissolved inorganic carbon, it was found that the highest $n$-pentadecane production rates occurred in samples from the deep chlorophyll maximum, where approximately $1 \%$ of photosynthetically active radiation penetrates [3]. This could be due to a number of factors. Hydrocarbons accumulate in cyanobacterial thylakoid membranes [4], and their abundance may increase as photosystem production is boosted to maximise light harvesting. It may also be related to the function of alkanes, which are speculated to increase membrane fluidity [4], potentially resulting in more alkane biosynthesis to counter membranes becoming inflexible in colder and deeper waters.

So, why aren't the oceans awash with hydrocarbons, in particular with cyanobacterial $n$ pentadecane? Diverse generalist and specialist bacterial species can use alkanes as a source of carbon and energy $[2 ; 5]$. Therefore, it may be expected that cyanobacterial $n$-pentadecane would sustain communities of alkane-degrading bacteria. Love et al. [3] provide evidence from the field to show that this is likely to be the case. When water from $500 \mathrm{~m}$ combined with particles collected from just beneath the deep chlorophyll maximum was incubated with $n$ pentadecane, oxygen consumption occurred relatively rapidly. In some cases there was an increase in the abundance of specialist alkane-degrading bacteria, such as Alcanivorax and Thalassolituus species. In other cases, catabolically more versatile genera were enriched, and their capacity for alkane degradation was identified from alkane-catabolism genes in metagenome assembled genomes [3]. This verified previous laboratory findings showing that marine hydrocarbon-degrading bacteria can consume cyanobacterial $n$-pentadecane [2]. In this process, the hydrocarbon-degrading bacteria release carbon dioxide, which in turn may be used by photoautotrophs. This has been called the short-term, or cryptic, hydrocarbon cycle (Fig. 1), as it likely occurs over a period of days and goes largely unseen.

What does this underappreciated component of the global carbon cycle mean for oil-spill response? Could it sustain large populations of hydrocarbon-degrading bacteria that multiply rapidly when oil is spilled at sea, thereby mitigating harm to ecosystems, communities and 
economies? Certainly, some of the microorganisms that were enriched in response to $n$ pentadecane are among the most abundant and active after an oil spill, such as the obligate hydrocarbon degraders that specialize in oxidizing medium/long-chain alkanes (e.g. Alcanivorax or Thalassolituus) [3; 5]. However, $n$-pentadecane would not enrich microorganisms specializing in the oxidation of polycyclic aromatic hydrocarbons (PAHs), which are prevalent during an oil spill, such as Cycloclasticus [5; 7]. Such metabolic niche partitioning is seen also in microbial consumption of medium/long-chain alkanes compared with short-chain alkanes [7]. Therefore, it was perhaps unsurprising that the authors found that open-sea communities, which are naturally supplied with cyanobacterial $n$-pentadecane, were not primed to degrade the volatile, 5 -carbon alkane, $n$-pentane. This was in contrast to microbial communities from environments where $n$-pentane seeps into the water column, which are therefore primed for its degradation [3]. Overall, it is likely that the short-term hydrocarbon cycle will select only for microbial communities capable of consuming medium or long-chain alkanes, which is important because they are a major component of most marine oil spills. However, it remains to be seen whether cyanobacteria-derived alkanes enrich for specialists, such as Alcanivorax and Thalassolituus, or whether they would be outcompeted by alkane-degrading generalists that consume a wider range of organic matter released after the death and lysis of phototrophs.

One of the most intriguing results from the current study was the possible contribution of Archaea to the cryptic hydrocarbon cycle [3]. Analysis of the Tara Oceans datasets identified an excess of genes coding for enzymes such as alkane monooxygenase (AlkB), which activates medium/long-chain alkanes including $n$-pentadecane, over those coding for the activation of short-chain alkanes and aromatic hydrocarbons. They also observed an abundance of yet-to-becultivated Euryarchaeal marine group II (MGII), possessing genes that are phylogenetically close to bacterial alkane monooxygenases [3]. One approach to determine the function of these putative alkane monooxygenases in the future would be to incubate MGII-rich seawater samples with environmentally relevant concentrations of $n$-pentadecane, for example using dynamic passive dosing [6]. Such enrichments could then be analysed using various approaches, including metatranscriptomics to see if putative alkB genes from MGII are preferentially expressed in the presence of slowly released alkanes. It would also be intriguing to clone and express an MGII alkB-like gene into a suitable host and determine whether it provides the ability to oxidize alkanes.

An important next step will be to consider eukaryotic microalgae, such as diatoms, which account for about $40 \%$ of marine primary production, especially in nutrient-rich coastal areas and higher latitudes [8]. The concentrations and types of hydrocarbons produced by different eukaryotic microalgae, including long-chain alkenes [9], coupled with measures of phytoplankton abundance across the oceans, will further enhance our understanding of the nature and extent of the cryptic hydrocarbon cycle. It will also be important to learn the extent to which this and other short-term hydrocarbon cycles between photoautotrophs and heterotrophs, such as cycling of the volatile hydrocarbon, isoprene [10], impact on global nutrient cycles. 


\section{Competing Interests}

The authors declare no competing interests.

\section{Figure legend}

Figure 1. Short-term and long-term hydrocarbon cycles in the global ocean. The long-term hydrocarbon cycle, on the right, generally occurs over millions of years. It involves diagenesis and catagenesis of organic matter, and the release (via seeps or spills) of petroleum containing thousands of different types of hydrocarbons, including alkanes, alkenes and polycyclic aromatic hydrocarbons (PAHs). In contrast, the short-term, or cryptic, hydrocarbon cycle, on the left, occurs over days. This timescale corresponds with the turnover time of the main marine photosynthetic cyanobacteria (determined to be 1.4 to 2.4 days [3], which agrees with previously published values of 1-2 days for Prochlorococcus and 1-6 days for Synechococcus), and the release of the medium/long-chain alkane, $n$-pentadecane (and to a lesser extent $n$-heptadecane) from lysed cells [2; 3]. The relative contribution of long-chain alkenes to the short-term hydrocarbon cycle, primarily from Eukaryotic phytoplankton [9], remains to be quantified. In both the longand short-term hydrocarbon cycle, hydrocarbon-degrading microorganisms play a central role. Those involved in the short-term cycle degrade medium/long-chain alkanes aerobically as a carbon and energy source, and consist of obligate hydrocarbon-degrading bacteria as well as more nutritionally versatile microorganisms, potentially including uncultivated Archaea such as MGII [3]. A much greater diversity of microorganisms is involved in degrading petroleum hydrocarbons, reflecting the variety of molecular structures and the locations where petroleum is found, including anoxic sediments.

\section{References}

1. National Research Council. Oil in the Sea III. doi:10.17226/10388 (2003) 
2. Lea-Smith, D.J. et al. Proc. Natl. Acad. Sci. USA 112, 13591-13596 (2015)

3. Love, C.R. et al. Nat. Microbiol. XX, XXX-XXX (2021)

4. Lea-Smith, D.J. et al. Plant Physiol. 172, 1928-1940 (2016)

5. McGenity, T.J. et al. Aquat. Biosyst. 8, 10 (2012)

6. Smith, K.E. et al. Environ. Sci. Technol. 46, 4852-4860 (2012)

7. McKew, B.A. et al. Environ. Microbiol. 9, 165-176 (2007)

8 Tréguer, P. et al. Nat. Geosci. 11, 27-37 (2018)

9. Sorigué, D. et al. Plant Physiol. 171, 2393-2405 (2016)

10. McGenity, T.J. et al. ISME J. 12, 931-941 (2018) 